\section{P156 ACCELERATED CORONARY ATHEROSCLEROSIS - A MAJOR CAUSE OF MYOCARDIAL INFARCTION IN SYSTEMIC LUPUS ERYTHEMATOSUS}

${ }^{1}$ Isak Samuelsson, ${ }^{1}$ loannis Parodis, ${ }^{1}$ Iva Gunnarsson, ${ }^{1}$ Agneta Zickert, ${ }^{2}$ Claes HofmanBang, ${ }^{2}$ Håkan Wallén, ${ }^{1}$ Elisabet Svenungsson. 'Division of Rheumatology, Dept. of Medicine Solna, Karolinska Institutet, Karolinska University Hospital, Stockholm; ${ }^{2}$ Dept. of Clinical Sciences, Division of Cardiovascular Medicine, Danderyd Hospital, Karolinska Institutet, Stockholm, Sweden

\subsection{6/lupus-2020-eurolupus. 198}

Background Patients with Systemic Lupus Erythematosus (SLE) are at increased risk of premature mortality due to myocardial infarction (MI). The underlying mechanisms are not fully understood. This study aims to generate new hypothesizes on these mechanisms through description of MI subtypes and locations and by identifying risk factors for MI.
Methods We identified 35 SLE patients with and a first-time non-procedural MI (MI-SLE). We matched these 35 MI-SLE patients to 35 patients with MI but not SLE (MI-nonSLE) and 35 patients with SLE but not MI (nonMI-SLE) for gender, age and geographical location. Patients and controls were matched individually $(1: 1: 1)$. Detailed retrospective medical file review was performed.

Results Median age was 62 years and $89 \%$ were female in all groups. Prevalence of ST-elevation MI was similar in MI-SLE patients and MI-nonSLE patients $(27 \%$ vs $36 \%$; $=0.80)$. The left ventricle was the most commonly infarcted in both MISLE and MI-nonSLE - $77 \%$ vs $59 \%$ according to coronary angiography and $42 \%$ vs $55 \%$ according to echocardiography. The left ventricular ejection fraction was similar in MI-SLE and MI-nonSLE patients $(p=0.62)$. MI with coronary atherosclerosis was trends wise more common in MI-SLE patients compared to MI-nonSLE patients (88\% vs 66\%; $\mathrm{p}=0.065)$. Previous cardiovascular disease $(43 \%, 5.7 \%, 14 \% ; \mathrm{p}<0.001)$, coronary artery disease $(31 \%, 2.9 \%, 2.9 \% ; \mathrm{p}<0.001)$ and low plasma albumin levels $(35 \mathrm{~g} / \mathrm{L}, 40 \mathrm{~g} / \mathrm{L}$, not determined; $\mathrm{p}=0.001$ ) distinguished MI-SLE patients from MI-nonSLE and nonMI-SLE patients.

Conclusion Coronary atherosclerosis was present in a large majority of MI-SLE patients at the event of MI. In addition, coronary artery disease preceding MI was more prevalent in SLE patients than in the general population, indicating accelerated coronary atherosclerosis as a cause of increased MI prevalence in SLE. Among SLE patients, low albumin levels were a risk factor for MI.

\section{P157 UPDATE ON THE REAL-TIME COLLECTING SYSTEM DATA OF SLE TREATMENT AND RESEARCH GROUP (CSTAR) REGISTRY: CLINICAL FEATURES AND REMISSION OF PATIENTS WITH SYSTEMIC LUPUS ERYTHEMATOSUS IN CHINA}

Ziqian Wang, Mengtao Li, Jiuliang Zhao, Qian Wang, Xiaofeng Zeng, CSTAR. Dept. of Rheumatology, Peking Union Medical College Hospital, Peking Union Medical College and Chinese Academy of Medical Sciences, Key Laboratory of Rheumatology and Clinical Immunology, Ministry of Education, Beijing, China

\subsection{6/lupus-2020-eurolupus.199}

Background A new real-time collecting system were set up from 2017 based on the first registry cohort in China. This study was an update on the clinical features and remission status of recent results.

Methods CSTAR registry was first launched in 2009. Based on CSTAR, a new mobile application program (APP) platform was established to collect real-time data in clinic with predesigned electronic case report form. All data were generated and uploaded in clinic directly without secondary collection, including demography, clinical manifestations, disease activity (SLEDAI-2K), organ damage (SLICC Damage Index), lab results, imaging and medications. Biological samples were preserved for future study at first clinic visit. Meanwhile, data cleaning and validation was managed by a professional backstage statistician.

Results A total of 22807 SLE patients from 295 different centers have been registered up to Sep 2019. Male to female rate was $1: 12$. The most common manifestations at entry were mucocutaneous (59.8\%), arthritis (55.3\%), hematologic $(37.9 \%)$ and lupus nephritis (34.6\%). In addition, neuropsychiatric disorder and pulmonary arterial hypertension took up 
for $5.4 \%$ respectively. 399 patients $(1.7 \%)$ had interstitial lung disease at baseline. The prevalence rate of coronary artery disease and cerebrovascular disease were both $0.7 \%$. The percentage of combined malignancy was $0.6 \%$. $57.6 \%$ of the patients were in remission at entry, and $19.6 \%$ of the patients had developed irreversible organ damage at baseline.

Conclusion The direct online reporting and collecting system of CSTAR had expanded with more than 20,000 patients involved all around China, showing us current situation of clinical practice for SLE in China and what we have achieved by applying Treat-To-Target strategy into daily practice for Chinese SLE patients.

Keywords Systemic Lupus Erythematosus, cohort, clinical features, remission

\section{\begin{tabular}{|l|l}
\hline P158 LUPUS MASTITIS: THE GREAT MIMMICKER \\
\hline
\end{tabular}}

${ }^{1}$ Rita Cunha, ${ }^{2}$ Alice Pimentel, 'Bernardo Figueiredo Santos, 'Renata Aguiar, ${ }^{2}$ Joana Noronha, ${ }^{1}$ Anabela Barcelos. ${ }^{1}$ Rheumatology Dept., Centro Hospitalar do Baixo Vouga, Aveiro; ${ }^{2}$ General Surgery Dept., Centro Hospitalar do Baixo Vouga, Aveiro, Portugal

10.1136/lupus-2020-eurolupus.200

Background Lupus Mastitis is rare and can be easily confounded with infection or breast cancer.

Methods We report two cases of lupus mastitis, in different clinical settings.

Results The first patient was a 34-year-old female with Systemic Lupus Erythematosus (SLE) with renal, hematological, musculoskeletal and cutaneous involvement, medicated with hydroxychloroquine (HCQ) $400 \mathrm{mg} / \mathrm{day}$, mycophenolate mofetil $2000 \mathrm{mg}$ /day and prednisolone $7.5 \mathrm{mg} /$ day. She presented to the emergency department with painful right breast. On the examination, the breast was swollen, warm and painful to palpation. Breast ultrasound revealed a vascularized and lobulated mass at the upper external quadrant, with liquid areas inside, suggesting possible abscess, which was drained and biopsied.

Histopathological analysis demonstrated a fibroinflammatory process, with fibrotic and abscedated areas, ductitis, lobulitis and vasculitis, compatible with lupus mastitis.

Cultures from the aspirated fluid were negative.

The patient was medicated with antibiotic, NSAIDs and prednisolone dose was increased to $10 \mathrm{mg} /$ day with significant improvement.

The second case refers to a 48-year-old female patient, with SLE with cutaneous, immunological and musculoskeletal involvement, who had withdrawn HCQ due to ocular toxicity. In the following months, the patient presented recurrent episodes of mastitis, on the same location. Breast ultrasound performed during one of the episodes revealed a hyperecogenic area of the fibroglandular tissue, with cystic areas. In spite of repeated treatment with antibiotics and NSAIDs, mastitis recurred, in a total of seven times. Because of worsening of the cutaneous lupus, the patient was medicated with methotrexate up to $15 \mathrm{mg} /$ week. No more episodes of mastitis were recorded and biopsy of the breast, which had been considered, was not performed due to total recovery. The gynecology and rheumatology teams concluded that lupus was the etiology of the recurrent mastitis.

Conclusions Clinicians should be aware of this entity to avoid unnecessary invasive procedures, which may increase inflammation involved in lupus mastitis. Therapeutic approach usually demands increasing immunomodulation.

\section{P159 \\ SIMILAR PROGRESSION OF CAROTID INTIMA-MEDIA THICKNESS IN 7-YEAR SURVEILLANCE OF PATIENTS WITH MILD SLE AND CONTROLS, BUT THIS PROGRESSION IN PATIENTS IS STILL PROMOTED BY DYSLIPIDEMIA, HYPERTENSION, HISTORY OF LUPUS NEPHRITIS AND A HIGHER PREDNISONE USAGE}

${ }^{1,2}$ Sofia Ajeganova, ${ }^{3}$ Thomas Gustafsson, ${ }^{3}$ Linnea Lindberg, ${ }^{1}$ Ingiäld Hafström, ${ }^{4}$ Johan Frostegåd. 'Dept. Medicine Huddinge, Karolinska Institutet, Stockholm, Sweden; ${ }^{2}$ Dept. Clinical Sciences, Vrije Universiteit Brussel, Brussels, Belgium; ${ }^{3}$ Dept. Laboratory Medicine, Karolinska Institutet, Stockholm; ${ }^{4}$ Dept. Environmental Medicine, Karolinska Institutet, Stockholm, Sweden

\subsection{6/lupus-2020-eurolupus.201}

Background Effect of classical risk factors on progression of subclinical atherosclerosis in patients with SLE in comparison with population controls is not clear. We aimed to compare progression of carotid intima-media thickness (cIMT) and factors promoting it in patients with SLE and controls.

Methods Patients with SLE and matched population controls from the SLEVIC-cohort were assessed at inclusion and after seven years with standardized data collection and carotid ultrasound. Effect of risk factors on cIMT progression was examined with adjusted linear mixed models.

Results A total of 77 patients and 74 controls, 68\% and $61 \%$ of the original cohort, completed follow-up. The patients were mean 47 years old, $90 \%$ females, controls were 51 years old, $92 \%$ females. Patients had disease duration of mean 11 years and mild disease activity. Baseline cIMT did not differ between the groups. An average absolute cIMT progression was $0.009 \mathrm{~mm} /$ year in patients and $0.011 \mathrm{~mm} /$ year in controls, intergroup difference $\mathrm{p}=0.9$. Dyslipidemia and hypertension at both assessments and carotid plaque at inclusion were associated with cIMT progression in patients and controls. History of lupus nephritis and a higher average dose of prednisolone used since diagnosis were associated with cIMT progression in patients. Associations of risk factors with cIMT progression was stronger in presence of plaques.

Conclusions We observed similar progression of cIMT in SLE and controls over 7 years, which implies that progression of subclinical atherosclerosis in some patients with SLE could be normalized. Traditional CV risk factors, history of lupus nephritis and higher use of corticosteroids promote cIMT progression in SLE. Detection of carotid plaque may add to CV risk stratification.

\section{P160 RISK OF CV EVENTS AND MORTALITY IN SLE IS ASSOCIATED WITH ACCUMULATED DISEASE-DAMAGE, ANTI-PHOSPHOLIPID SYNDROME AND HIGHER CAROTID INTIMA-MEDIA THICKNESS}

${ }^{1,2}$ Sofia Ajeganova, ${ }^{1}$ Ingiäld Hafström, ${ }^{3}$ Johan Frostegåd. ${ }^{1}$ Dept. Medicine Huddinge,
Karolinska Institutet, Stockholm, Sweden; ${ }^{2}$ Dept. Clinical Sciences, Vrije Universiteit Brussel,
Brussels, Belgium; ${ }^{3}$ Dept. Environmental Medicine, Karolinska Institutet, Stockholm, Sweden

10.1136/lupus-2020-eurolupus.202

Background SLE is a strong risk factor for premature CVD and mortality. We investigated which factors could explain poor prognosis in SLE compared with controls.

Methods Patients with SLE and age- and sex-matched controls were recruited for this prospective study. Carotid ultrasound was performed at inclusion. The outcome was 